Punjab University Journal of Mathematics (2021),53(8)593-606

https://doi.org/10.52280/pujm.2021.530805

\title{
Semi-analytical solutions for the hydrodynamic stability based nonlinear fourteenth order differential problem
}

\author{
Islam Zari \\ Jinnah College for Women, \\ University of Peshawar, Khybar Pakhtunkhwa, 25000, Pakistan. \\ Email: zarimaths@uop.edu.pk \\ Tahir Saeed Khan \\ Department of Mathematics, \\ University of Peshawar, Khybar Pakhtunkhwa, 25000, Pakistan. \\ Email: tsk7@uop.edu.pk \\ Fahim Ullah \\ Department of Electrical Engineering, \\ University of Science and Technology Bannu, Khybar Pakhtunkhwa, 28100, Pakistan. \\ Email: fahimullah16151@gmail.com
}

Received: 17 July, 2020 / Accepted: 12 June, 2021 / Published online: 26 August, 2021

\begin{abstract}
This research article is concerned with the solution of hydrodynamic stability based linear and nonlinear fourteenth order differential problem, which has great significance in applied physics, astrophysics, applied mathematics, engineering departments. The homotopy perturbation method (HPM) and optimal homotopy asymptotic method (OHAM) are applied for the solution of the existed problem. These semi analytical techniques are continuously evolved to solve diverse range of linear and nonlinear problems with effective approximate agents which is a rapid approach to the exact solutions. This approach is effectively proposed with different numerical examples, which are taken from literature. Numerical results are accomplished by phrase of convergent series solutions and approach to the accurate solutions only by taking minimum steps. The numerical results are exercised with exact solutions, cubic polynomial spline technique (CPST) and cubic non-polynomial spline technique (CNPST), excellent agreement has been observed. The observations suggested that OHAM and HPM performed excellent in comparison to the CPST and CNPST in terms of solution, which demonstrated the effectiveness, potential and validity of suggested schemes in reality and acquired results are of top-level perfection.
\end{abstract}


AMS (MOS) Subject Classification Codes: 35S29; 40S70; 25 U09

Key Words: Fourteenth order problem; optimal homotopy asymptotic technique; homotopy perturbation technique; approximate solutions.

\section{INTRODUCTION}

Fluid flow analysis comprising various boundary conditions is of utmost interest; especially for the last few decades, it has gained importance among researchers and engineers. It has diversified applications in all sections of science and engineering, such as in the field of industrial system models and analysis, applied physics, aerospace analysis, and environmental engineering. Due to its significance, a considerable practice is continued to attain the estimated illustrations theoretically for rigorously endeavor, problematic flow models with low computational cost. For instance, one of existed complex stability based boundary value problems (BVPs) is introduced in the field of hydrodynamic and hydro- magnetic. The hydrodynamic theory related to the stationary solutions, which is applied in elementary flow patterns between objects such as rotating cylinders and parallel planes. However, the restriction of these flow patterns is identified by ranges of parameters. As a result, hydrodynamic stability problems developed from unsteady pattern of authorized flows. On the other hand, hydro-magnetic study is based on hydrodynamic flows of electrically governing liquids with magnetic fields. In fact, these types of boundary layer flows, which have great issues of stability can not be considered without a magnetic field. Earlier, researchers have been exploring significant contribution to overcome such type of problems, related to the solution of various boundary value conditions over physical issues and now this phenomenon is also concerned with partial differential equations, which has a main focus to considerable a general direction. In this regard, Derakhshan et al. [17] examined the hydrothermal behavior of magneto hydrodynamic based nanofluid flow between two parallel disk utilizing the AGM. Chamkha et al. [16], revealed magneto related hydrodynamic flow and analyzed the transfer of heat rate in hybrid nanofluid towards two surfaces with the aid of thermal radiation and Joule heating, respectively. Further, multiple and various boundary value conditions are continuously applied on partial differential equations scrutinized in (see, for instance, [10]-[21]).

To tackle such type of interesting problems, most of researchers focused their attention to utilize a plethora of numerical and analytical techniques to overcome different ordered linear and nonlinear differential issues, which are effective in nature throughout literature survey reports (see, for example, [7]-[39]). The reason behind this approach is regular application in applied mathematics and engineering departments like astrophysics, beam theory and hydrodynamic and hydro magnetic stability analysis. In fact, these methods are helpful and also applied for production of daily life use advanced items such as aerospace section and to resolve diseases for health improvement, by which the internal influences of various systems can be observed. In view of their importance, Barari et al. [13] presented the HPM for the solution of tenth order differential problems. Ali et al. [5] attempted the special type of twelfth order differential problems and solved via OHAM. Haq et al. [20] applied the OHAM to eighth order differential problems. Ali et al. [6] addressed the 
solution of sixth order parameterized differential problem utilizing OHAM. Nawaz et al. [38] simulated the tenth order boundary value problems utilizing the OHAM procedure. Similarly, HPM mechanism is followed by Saberi and Zahmatkesh [40] to solve higher order differential problems. Mohyud-Din and Yildirim [33] explored the solution of ninth and tenth order differential problems by HPM.

Although, a huge number of different methods have been used previously for some linear and nonlinear differential problems, there is still need to construct methods which are accurate and of low computational cost for solutions of dynamic obstacles. The solution of these types of physical problems, one-step hybrid block method has been numerically applied by Abdelrahim et al. [1] to solve the third order differential problems and results signified that this developed scheme is capable to generate better outcomes while comparable to previous literature. Homotopy type techniques such as OHAM and HPM are applied for solution of higher order BVPs by Naeem et al. [35]. Arora et al. [9] examined a numerical scheme for higher order BVPs. Ghomanjani and Shateyi [18] addressed the alternative mathematical procedures for the solution of two point nonlinear boundary value problems. Appadu and Kelil [8] worked on the semi-analytic methods for the solution of linearized dispersive KdV equations. Shafiq et al. [41] highlighted the statistical approach on the stagnation point nanofluid flow over radiative Riga surface utilizing the semi-analytical technique OHAM. Ayati and Ahmady [11] examined the comparison of semi-analytical techniques via OHAM and HPM to solve the Fisher equation. Some other interesting study has been done for the solution of differential problems by OHAM can be seen in the literature (see, for instance, [3]-[34]).

In OHAM and HPM, the homotopy concept comes from conventional perturbation method and topology, which are packed with the introduction of an analytical procedure to overcome the linear and nonlinear differential problems. These are simple and straightforward, which does not require any existence of small or large parametric value for the strongly nonlinear differential problems as does the classical perturbation techniques. Unlike some other homotopy techniques, OHAM certify a quick convergence, however it requires only few terms to reach an accurate solution. In OHAM, the convergence area can be simply managed and adjusted. In fact, OHAM gives an easy idea to control the convergence area for strongly nonlinear problems and is successfully applied to such type of problems arising in fluid mechanics and heat transfer as studied in ([29]- [30]). In comparison to OHAM, the HPM is also easy to interpret, can find highly accurate solutions in just small number of iterations [27] with low computational cost as compare to OHAM and eliminated the limitations of traditional perturbation methods. In this direction, Roslen et al. [?] investigated the analytical comparison for the solution of an upper-convected Maxwell MHD fluid flow over a porous channel. Gupta and Ray [19] judged the comparison between HPM and OHAM for the solutions of Boussinesq related Burger system. Although, the convergence area of the acquire truncated series solution is restricted and is the excellent case scenario, it requires some improvements to expand the convergence area of the approximate result. Therefore, He in [22] extended this technique of nonlinear analysis for a wide range of problems. Hence, in view of literature survey (see, for example, [44]-[2]), two semi-analytical schemes OHAM and HPM are flexible, effective in terms of error analysis for higher ordered boundary value differential problems. 
A major extension of the present research is to solve the hydrodynamic stability based fourteenth order linear and nonlinear differential problem via OHAM and HPM. To the best of our knowledge, such a study does not previously attempted by utilizing any semianalytical techniques.

The general type of fourteenth order differential equation structure is given as (see, for instance, [37]):

$$
\begin{array}{r}
\frac{d^{14} \check{p}}{d \tilde{t}^{14}}=g(v, \check{p}(z)), c \leq v \leq d . \\
\check{p}^{(2 i)}(c)=h_{i}, \check{p}^{(2 i)}(d)=l_{i}, \\
h_{i}, l_{i}=0,1,2,3,4,5,6 .
\end{array}
$$

\section{AnAlytical Schemes}

2.1. Basic theme of OHAM. The concept of this method is based on homotopy and it was introduced for the first time in 2008 with by effort of Herisanu and Marinca ([32][31]). The description of the method for the ordinary boundary value problem is followed as:

$$
A(z(x))+f(x)+B(z(x))=\zeta, M\left(z(x), \frac{d z}{d x}\right),
$$

where $A$ and $B$ signify the linear and nonlinear operators, $z(x)$ shows independent variable in an unspecified function, $f(x)$ represents a specified function and $M$ manifests boundary operator.

An equation of bend equations is created which can be suggested as:

$$
\begin{array}{r}
(1-\delta)[A(\check{\psi}(x, \delta))+f(x)]= \\
T(r)[A(\check{\psi}(x, \delta))+f(x)+B(\check{\psi}(x, \delta))] M\left(\check{\psi}(x, \delta), \frac{\partial \check{\psi}(x, \delta)}{\partial x}\right)=\zeta,
\end{array}
$$

where $\delta \in[0,1]$ presents an inserting variable, the approach of the general result is dependent on $T(\delta)$ indicates a convergence of general solution, which is based on an auxiliary function.

The approximate solution of $\check{\psi}(x, \delta)$ is represented of $\delta$ by utilizing the Taylor series,

$$
\check{\psi}\left(x, \delta, A_{k}\right)=z_{0}(x)+z_{1}\left(x, A_{1}\right) \delta^{1}+z_{2}\left(x, A_{2}\right) \delta^{2}+\ldots
$$

The convergence rate of the equation (2. 5) is very efficient in terms of solution, which is devoted with auxiliary constants $A_{i}$. Further, constants converge, then obtained equation can be written as:

$$
z\left(x, A_{1}, A_{2}, \ldots, A_{m}\right)=z_{0}(x)+\sum_{k=1}^{\infty} z_{k}\left(x, A_{1}, A_{2}, \ldots, A_{m}\right) .
$$

Inserting an equation (2. 5) into the equation (2. 2), the updated form of the equation in terms of residual is:

$$
Q\left(x, A_{1}, A_{2}, \ldots, A_{m}\right)=A\left(z\left(x, A_{1}, A_{2}, \ldots, A_{m}\right)\right)+B\left(z\left(x, A_{1}, A_{2}, \ldots, A_{m}\right)\right) .
$$


If $Q\left(x, A_{1}, A_{2}, \ldots, A_{m}\right)=\zeta$, then $z$ is presented exact solution. Generally, these constraints are changed for nonlinear problems. The values of $A_{1}, A_{2} \ldots$ are attained by utilizing least square method. Moreover, approximate solution is acquired by putting these constants in equation (2.6).

2.2. Main theme of HPM. The basic idea of this technique is presented by taking nonlinear boundary value problem stated as:

$$
G(\check{m})=g(i) ; i \in \Omega, M\left(\check{m}, \frac{\partial \check{m}}{\partial s}\right), i \in J .
$$

Here, $G(\check{m})$ signifies differential operator, $M$ indicates a boundary operator, $g(i)$ stands for an analytical function, $\Omega$ presents the domain of boundary. Further, consider the equation (2. 8), which may be rephrased as:

$$
P(\check{m})+B(\check{m})=g(i) .
$$

After inserting a homotopy

$$
\alpha(i, e): \Omega \times[0,1] \longrightarrow R
$$

which satisfies

$$
M(\alpha, e)=(1-e)\left[\left(P(w)-H\left(b_{0}\right)\right]+e[G(\alpha)-g(i)]=0,\right.
$$

where $e \in[0,1]$ is an embedding parameter. Suppose the primary estimation is $b_{0}$ for equation (2. 8). The resulting series solution can be obtained by applying the homotopy procedure on equation (2.11), which can be represented as:

$$
w=w_{0}+e w_{1}+e^{2} w_{2}+e^{3} w_{3}+\ldots
$$

If $e \longrightarrow 1$, then the equation (2. 12) will converge to approximate solution and converted to equations (2. 13), i.e.

$$
\check{m}(x)=\lim _{e \longrightarrow 1} w=w_{0}+w_{1}+w_{2}+w_{3}+\ldots
$$

\section{Methods Application}

3.0.1. OHAM solution for model 1. Consider the linear fourteenth order problem [37]:

$$
\frac{d^{14} \check{p}}{d t^{14}}=-\cos (t)-\sin (t), 0 \leq t \leq 1,
$$

the boundary conditions are:

$\check{p}^{(2 k)}(0)=1, \check{p}^{(2 k)}(1)=\cos (1)+\sin (1)$,

$$
\check{p}^{(2 l)}(0)=-1, \check{p}^{(2 l)}(1)=\cos (1)-\sin (1),
$$

where $k=0,4,8,12$ and $l=2,6,10$.

The exact value of the above problem is $\check{p}(t)=\cos (t)+\sin (t)$.

After applying OHAM on an equation (3. 14), we get,

Zeroth order problem deformation:

$$
\check{p}_{0}^{(14)}(t)=0
$$


the series solution is:

$$
\begin{aligned}
& \check{p}_{0}(t)=1+t-0.5 t^{2}-0.16666666666666666 t^{3}+0.041666666666666664 t^{4} \\
& +0.008333333333333333 \mathrm{t}^{5}-0.001388888888888889 t^{6}-0.00019841271417284502 t^{7}+ \\
& 0.000024801695545336853 t^{8}+2.7554108328333 \times 10^{-6} t^{9}-2.7504574973136187 \times \\
& 10^{-7} t^{10} \\
& -2.557538788427006 \times 10^{-8} t^{11}+2.3944711042975737 \times 10^{-9} t^{12} \\
& +6.32942355812229 \times 10^{-11} t^{13} .
\end{aligned}
$$

First order problem deformation:

$$
\check{p}_{1}^{(14)}\left(t, A_{1}\right)=\left(1+A_{1}\right) \check{p}_{0}^{(14)}(t)+A_{1}[\cos (t)-\sin (t)]
$$

then the solution is:

$$
\begin{aligned}
& \quad \check{p}_{1}(t)=A_{1}+t A_{1}-0.5 t^{2} A_{1}-0.16666666666666666 t^{3} A_{1}+0.0416666664 t^{4} A_{1} \\
& \quad+0.008333333333333333 \mathrm{t}^{5} A_{1}-0.001388888888888889 t^{6} A_{1}-0.00019841271455334208 t^{7} A_{1} \\
& +0.000024801700419629924 t^{8} A_{1}+2.7554124244488776 \times 10^{-6} t^{9} A_{1}-2.750384737737477 \times \\
& 10^{-7} t^{10} A_{1}-2.5585904950276017 \times 10^{-8} t^{11} A_{1}+2.396518539171666 \times 10^{-9} t^{12} A_{1} \\
& +6.30961949354969 \times 10^{-11} t^{13} A_{1}-\sin (t) A_{1}-\cos (t) A_{1} .
\end{aligned}
$$

After applying equation (3. 16) and equation (3. 18), for $r=1$, the first order approximate value via $\mathrm{OHAM}$ is:

$$
\check{p}\left(t, A_{1}\right)=\check{p}_{0}(t)+\check{p}_{1}\left(t, A_{1}\right)
$$

To obtain unknown convergent constants, we used the least square mechanism. Hence, by switching $A_{1}$ value in equation (3. 19), we attained second order approximation.

3.0.2. OHAM solution for model 2. Consider the nonlinear fourteenth order differential problem [37]:

$$
\frac{d^{14} \check{p}(t)}{d t^{14}}=e^{-t} \check{p}^{2}(t), 0 \leq t \leq 1
$$

subject to boundary conditions:

$\check{p}^{(2 k)}(0)=1, \check{p}^{(2 k)}(1)=e$,

where $k=0,1,2,3,4,5,6$.

The exact value of the above problem is $\check{p}(t)=e^{t}$.

After utilizing the OHAM procedure for the equation (3. 20), we get

Zeroth order problem deformation:

$$
\check{p}_{0}^{(14)}=0
$$


The terms of solution are:

$$
\check{p}_{0}(t)=\frac{1}{720}\left(720+720 t+360 t^{2}+120 t^{3}+\right.
$$

$30 \mathrm{t}^{4}+6 t^{5}+t^{6}-2095015 t^{7}+770713 e t^{7}+10872513 t^{8}-3999774 e t^{8}-23956775 t^{9}+$ $8813205 e t^{9}+28561259 t^{10}-10507100 e t^{10}-19373181 t^{11}+7126995 e t^{11}+7073725 t^{12}-$ $\left.2602278 e t^{12}-1084483 t^{13}+398959 e t^{13}\right)$.

First order problem deformation is:

$$
\check{p}_{1}^{(14)}(t)=\left(1+A_{1}\right) \check{p}_{0}^{(14)}(t)+e^{-t} A_{1} \check{p}_{0}^{3}(t) .
$$

The series solution can be considered as:

$$
\begin{aligned}
& \check{p}_{1}\left(t, A_{1}\right)=\frac{1}{518400} e^{(-1-t)}\left(-2748614852766544374072 . .51440262400 e A_{1}\right. \\
& +2022317792063097915881842944620543053949399040000 \mathrm{e}^{2} A_{1} \\
& -371984569607716780208001336282338563575536640000 e^{3} A_{1} \\
& +2748614852766544374072695780222842152551440262400 e^{1+t} A_{1} \\
& -2022317792063097915881842944620543053949399040000 e^{2+t} A_{1} \\
& +371984569607716780208001336282338563575536640000 e^{3+t} A_{1} \\
& \text { - 1840556071740181936810764183918928887383948044800et } A_{1} \\
& +1354205478233321035191366080981727532516876800000 e^{2} t A_{1} \\
& \text { - } 249092177281955189499375601074197144841922560000 e^{3} t A_{1} \\
& \text { - 908058781026362437261931596303913265167492217600e } e^{1+t} t A_{1} \\
& +668112313829776880690476863638815521432522240000 e^{2+t} t A_{1} \\
& -122892392325761590708625735208141418733614080000 e^{3+t} t A_{1} \\
& \text { - 608282937037822313677945046730432587340646924800et }{ }^{2} A_{1} \\
& +447549573903311834463080598598218298927403520000 e^{2} t^{2} A_{1} \\
& \text { - 82322143572055155999007042732958645809374720000e } e^{3} A_{1} \\
& +142034291680912563903528752922924776232419011200 e^{1+t} t^{2} A_{1} \\
& \text { - ..-20802248294484940104415541825779et } t^{16} A_{1}+15305438955372874531313547658500 e^{2} t^{16} A_{1} \\
& -2815278164893734342015890283236 e^{3} t^{16} A_{1}-538970680429221804997307250074 e t^{17} A_{1} \\
& +396552465448276438229439000274 e^{2} t^{17} A_{1}-72941749692151027822818176720 e^{3} t^{17} A_{1} \\
& -12424581840147590626290184071 e t^{18} A_{1}+9141496448286689985917673990 e^{2} t^{18} A_{1} \\
& -1681484302433583599679444447 e^{3} t^{18} A_{1}-253038994594087339115260848 e t^{19} A_{1} \\
& +186175687851752867940685044 e^{2} t^{19} A_{1}-34245104003313210247175112 e^{3} t^{19} A_{1} \\
& -4505390806405133577234357 e t^{20} A_{1}+3314881304239245776010804 e^{20} t^{20} A_{1} \\
& \text { - 609738340876726000887030e } e^{30} t^{20} A_{1}-69064981266450392509842 e t^{21} A_{1} \\
& +\ldots+303691071318318 e^{2} t^{25} A_{1}-55860850802680 e^{3} t^{25} A_{1} \\
& \left.-1176103377289 e t^{25} A_{1}+865328506394 e^{2} t^{25} A_{1}-159163681 e^{3} t^{26} A_{1}\right) \text {. }
\end{aligned}
$$

After utilizing equation (3. 22) and equation (3. 24), for $r=1$, the first order approximate value via OHAM is:

$$
\check{p}\left(t, A_{1}\right)=\check{p}_{0}(t)+\check{p}_{1}\left(t, A_{1}\right) .
$$

Now reach to unknown convergent constants, the least square scheme is used for $\tilde{\tilde{p}}$. By putting value of $A_{1}$ in equation (3.25), the first order series solution is derived. 
3.0.3. HPM solution for model 1. Consider the fourteenth order differential problem [37]:

$$
\frac{d^{14} \check{p}}{d t^{14}}=-\cos (t)-\sin (t), 0 \leq t \leq 1
$$

subject to boundary conditions:

$\check{p}^{(2 k)}(0)=1, \check{p}^{(2 k)}(1)=\cos (1)+\sin (1)$,

$\check{p}^{(2 l)}(0)=-1, \check{p}^{(2 l)}(1)=\cos (1)-\sin (1)$,

where $k=0,4,8,12$ and $l=2,6,10$.

The exact value of the problem is $\check{p}(t)=\cos (t)+\sin (t)$.

After applying the HPM scheme on equation (3. 26), we can get:

Zeroth order problem deformation:

$$
\check{p}_{0}^{(14)}(t)=0
$$

Zeroth order solution is:

$$
\begin{gathered}
\check{p}_{0}(t)=1+t-0.5 t^{2}-0.16666666666666666 t^{3}+0.041666666666666664 t^{4} \\
+0.008333333333333333 \mathrm{t}^{5}-0.001388888888888889 t^{6}-0.00019841271417284502 t^{7}+ \\
0.000024801695545336853 t^{8}+2.755410832833310^{-6} t^{9}-2.750457497313618710^{-7} t^{10} \\
-2.55753878842700610^{-8} t^{11}+2.394471104297573710^{-9} t^{12}+6.3294235510^{-11} t^{13} \text {. (3. 28) }
\end{gathered}
$$

First order problem deformation:

$$
\check{p}_{1}^{(14)}(t)=\cos (t)-\sin (t)
$$

The solution is:

$$
\check{p}_{1}(t)=\frac{1}{-720}\left(-720-720 t+360 t^{2}+120 t^{3}-30 t^{4}-6 t^{5}+t^{6}+\right.
$$

$1711961 \mathrm{t}^{7}$

$-8967351 t^{8}+19888705 t^{9}-23827189 t^{10}+16223139 t^{11}-5941391 t^{12}+913121 t^{13}-$ $561911 t^{7} \cos (1)+2854962 t^{8} \cos (1)-6183975 t^{9} \cos (1)+7269940 t^{10} \cos (1)-4874085 t^{11} \cos (1)+$ $1762266 t^{12} \cos (1)-267917 t^{13} \cos (1)+720 \cos (t)-1673687 t^{7} \sin (1)+8823606 t^{8} \sin (1)-$ $19664955 t^{9} \sin (1)+23648140 t^{10} \sin (1)-16149885 t^{11} \sin (1)+5929182 t^{12} \sin (1)-$ $\left.913121 t^{13} \sin (1)+720 \sin (t)\right)$.

Using equation (3. 28) and equation (3. 30), we attained solution which is:

$$
\check{p}(t)=\check{p}_{0}(t)+\check{p}_{1}(t)
$$

3.0.4. HPM solution for model 2. Consider the fourteenth order nonlinear differential problem esteemed as [37]:

$$
\frac{d^{14} p(t)}{d t^{14}}=e^{-t} \check{p}^{2}(t), 0 \leq t \leq 1
$$


TABLE 1. Comparison of OHAM and HPM absolute error with CPST and CNPST absolute error [37] for model 1.

\begin{tabular}{|c|c|c|c|c|c|}
\hline$t$ & Exact & $\mid$ Exact-OHAM $\mid$ & $\mid$ Exact-HPM $\mid$ & $\mid$ Exact-CPST $\mid$ & $\mid$ Exact-CNPST $\mid$ \\
\hline & & (Current) & (Current) & {$[37]$} & [37] \\
\hline 0.2 & 1.1787359 & 0 & $2.2204 \times 10^{-16}$ & $3.77 \times 10^{-04}$ & $6.18 \times 10^{-07}$ \\
\hline 0.4 & 1.3104793 & $3.5527 \times 10^{-15}$ & $1.9984 \times 10^{-15}$ & $5.84 \times 10^{-04}$ & $9.47 \times 10^{-07}$ \\
\hline 0.6 & 1.3899780 & $9.7256 \times 10^{-14}$ & $5.8397 \times 10^{-14}$ & $5.94 \times 10^{-04}$ & $9.68 \times 10^{-07}$ \\
\hline 0.8 & 1.4140628 & $9.5901 \times 10^{-13}$ & $8.1801 \times 10^{-13}$ & $3.98 \times 10^{-04}$ & $6.60 \times 10^{-07}$ \\
\hline
\end{tabular}

TABLE 2. Comparison of OHAM and HPM absolute error with CPST and CNPST absolute error [37] for model 2.

\begin{tabular}{|c|c|c|c|c|c|}
\hline$t$ & Exact & $\mid$ Exact-OHAM $\mid$ & $\mid$ Exact - HPM $\mid$ & $\mid$ Exact - CPST $\mid$ & $\mid$ Exact - CNPST $\mid$ \\
\hline & & (Current) & (Current) & {$[37]$} & {$[37]$} \\
\hline 0.2 & 1.221403 & 0 & 0 & $3.71 \times 10^{-04}$ & $5.57 \times 10^{-07}$ \\
\hline 0.4 & 1.4918247 & $1.3323 \times 10^{-15}$ & $1.5543 \times 10^{-15}$ & $5.92 \times 10^{-04}$ & $7.24 \times 10^{-07}$ \\
\hline 0.6 & 1.8221188 & $1.1279 \times 10^{-13}$ & $1.2546 \times 10^{-13}$ & $6.35 \times 10^{-04}$ & $6.42 \times 10^{-07}$ \\
\hline 0.8 & 2.2255409 & $1.9096 \times 10^{-12}$ & $9.3259 \times 10^{-15}$ & $4.59 \times 10^{-04}$ & $3.84 \times 10^{-07}$ \\
\hline
\end{tabular}

subject to boundary conditions:

$$
\check{p}^{(2 k)}(0)=1, \check{p}^{(2 k)}(1)=e,
$$

where $k=0,1,2,3,4,5,6$.

The exact value of the problem is $\check{p}(t)=e^{t}$.

By applying HPM on equation (3. 32), we get

Zeroth order problem deformation:

$$
\check{p}_{0}^{(14)}(t)=0
$$

The solution is:

$$
\check{p}_{0}(t)=\frac{1}{720}\left(720+720 t+360 t^{2}+120 t^{3}+30 t^{4}+6 t^{5}+t^{6}-\right.
$$

$-2095015 \mathrm{t}^{7}+770713 e t^{7}+10872513 t^{8}-3999774 e t^{8}-23956775 t^{9}+8813205 e t^{9}$

$+28561259 t^{10}-10507100 e t^{10}-19373181 t^{11}+7126995 e t^{11}+7073725 t^{12}$ 


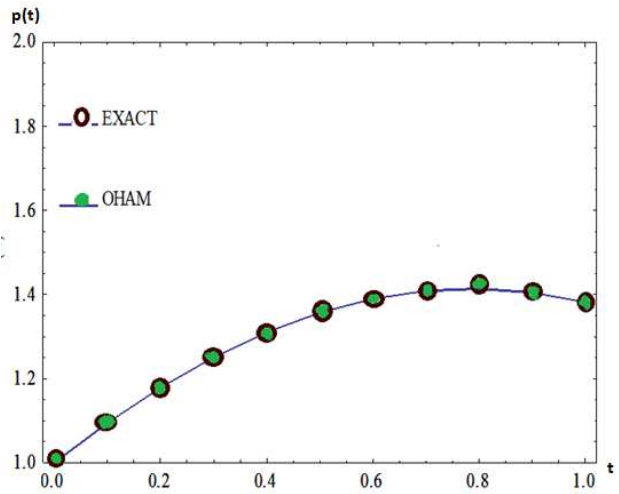

FIGURE 1. Significance of exact solution with OHAM solution for model 1 .

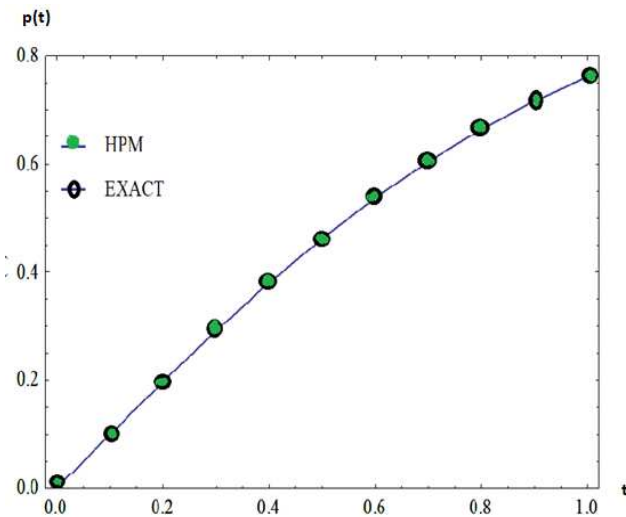

FIGURE 2. Significance of exact solution with HPM solution for model 2.

$$
\left.-2602278 e t^{12}-1084483 t^{13}+398959 e t^{13}\right) .
$$

Using equation (3. 34), then final solution is:

$$
\check{p}(t)=\check{p}_{0}(t) .
$$




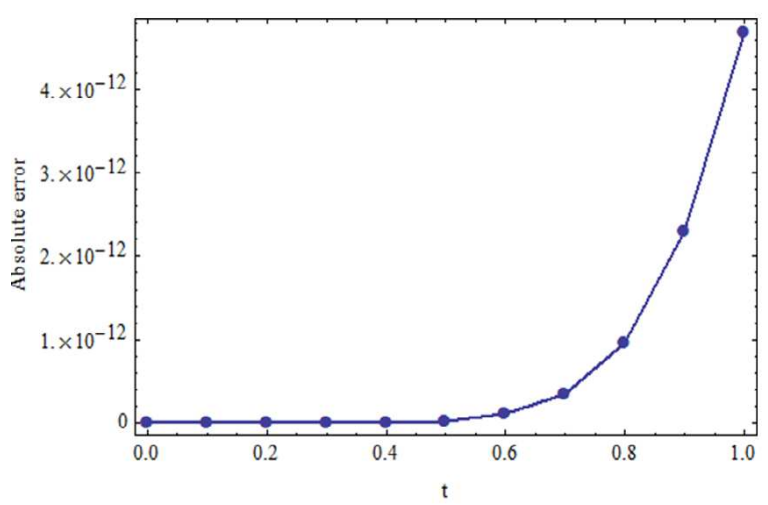

FIGURE 3. Plot of absolute error vs t using OHAM for model 1.

\section{RESULTS REPRESENTATION}

This section is committed to demonstrate the comparison of our proposed methods, results with other mathematical schemes in terms of tables and figures. A comparison of exact solution with OHAM solution, HPM solution, CPST solution, CNPST solution is demonstrated via absolute errors. Overall, OHAM and HPM solutions show excellent quality solutions with low computational cost for both problems while in comparison to other schemes. In Table 1, OHAM and HPM performed efficiently than cubic polynomial spline and cubic non-polynomial spline absolute error solution for the model 1. Similarly, in Table 2, high performance of same proposed methods observed while comparing to others schemes by taking various terms of $t=0.2,0.4,0.6,0.8$ for model 2 . Figure 1 , illustrated the best quality of OHAM solution, which is compared to the exact solution for model 1. On similar way, Figure 2, is depicted the comparison of exact solution with HPM for problem 2, which signifies very close values of the exact solution. Further, Figures 3-4 illustrated the absolute errors via OHAM and HPM.

\section{CONCLUSION}

In this paper, we adopted two new schemes known as OHAM and HPM for solution of hydrodynamic-magnetic stability based linear and non-linear fourteenth order differential equations. These methods have successfully been applied on two models for numerical testing, investigated more accurate and efficient results than other analytical methods and also provides the vivid agreement to exact solutions in terms of series solution. The suggested schemes have great potential to solve any type of linear and nonlinear problems and also demanding for less iteration to get accurate results, which are pros of these methods in the application field of industry and engineering departments. In fact, the fast convergence of applied techniques demonstrated the principal potency of OHAM and HPM, which is proved by comparing analytical solutions with other known methods. 


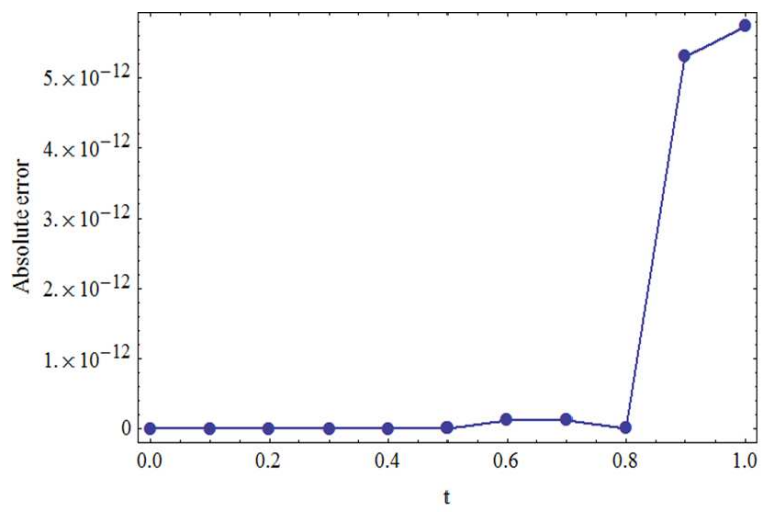

FIGURE 4. Plot of absolute error vs t using HPM for model 2.

\section{ACKNOWLEDGMENTS}

We thank the respected anonymous reviewers who contributed towards the improvement of this paper.

\section{REFERENCES}

[1] R. F. Abdelrahim, Numerical solution of third order boundary value problems using one-step hybrid block method, Ain Shams Eng. J. 10, No. 1 (2019) 179-183.

[2] W. Aibarakati, A. R. Seadaw, N. H. Aljahdaly, Application of mathematical methods for the nonlinear seventh order Sawada-Kotera Ito dynamical wave equation, Thermal Sci. (2019) 373-373.

[3] P. Agarwal, M. Akbar, R. Nawaz, M. Jleli, Solutions of system of Volterra integro-differential equations using optimal homotopy asymptotic method, M. Methods App. Sci. 44, No. 3 (2021) 2671-2681.

[4] S. Ahsan, R. Nawaz, M. Akbar, K. S. Nisar, E. E. Mahmoud, M. M. Alqarni, Numerical solution of 2Dfuzzy Fredholm integral equations using optimal homotopy asymptotic method, Alexandria Eng. J. 60, No. 2 (2021) 2483-2490.

[5] J. Ali, S. Islam, M. T. Rahim, G. Zaman, The solution of special twelfth order boundary value problems by the optimal homotopy asymptotic method, W. App. Sci. J. 11, No. 3 (2010) 371-378.

[6] J. Ali, S. Islam, H. Khan, G. Zaman, The solution of a paramterized sixth order boundary value problem by the Optimal homotopy asymptotic method, Pro. Romanian Aca. S. A. 12, No. 3 (2011) 167-172.

[7] M. A. AL-Jawary, G. H. Radhi, J. Ravnik, Daftardar-Jafari method for solving nonlinear thin film flow problem, Arab J. Basic Appl. Sci. 25, No. 1 (2018) 20-27.

[8] A. R. Appadu, A. S. Kelil, On Semi-Analytical Solutions for Linearized Dispersive KdV Equations, Mathematics, 8, No. 10 (2020) 1769.

[9] G. Arora, R. Kumar, H. Kaur, A novel wavelet based hybrid method for finding the solutions of higher order boundary value problems, Ain Shams Eng. J. 9, No. 4 (2018) 3015-3031.

[10] I. S. Awaludin, A. Ishak, I. Pop, On the stability of MHD boundary layer flow over a stretching/shrinking wedge, Sci reports, 8, No. 2 (2018) 13622.

[11] Z. Ayati, S. Ahmady, The comparison of OHAM and HPM to solve Fisher equation, Comp. Methods Diff. Eqs. 4 No. 1 (2016) 43-53.

[12] A. Babaei, S. Banihashemi, A. Mohammadpour, A Numerical Scheme to Solve an Inverse Problem Related to a Time-Fractional Diffusion-Wave Equation with an Unknown Boundary Condition, Punjab Univ. J. math. 51, No. 2 (2020).

[13] A. Barari, M. Omidvar, T. Najafi, A. R. Ghotbi, Homotopy perturbation method for solving tenth order boundary value problems, I. J. Maths. Comp. 3, J09 (2009) 15-27. 
[14] T. Batool, M. Ozair Ahmad, Application of Bernstein Polynomials for Solving Linear Volterra IntegroDifferential Equations with Convolution Kernels, Punjab Univ. J. math. 49, No. 3 (2020).

[15] M. El-Gamel, W. Adel, Numerical investigation of the solution of higher-order boundary value problems via Euler matrix method, Sema J. 75, No. 2 (2018) 349-364.

[16] A. J. Chamkha, A. S. Dogonchi, D. D. Ganji, Magneto-hydrodynamic flow and heat transfer of a hybrid nanofluid in a rotating system among two surfaces in the presence of thermal radiation and Joule heating, AIP Advances, 9, No. 2 (2019) 025103.

[17] R. Derakhshan, A. Shojaei, K. Hosseinzadeh, M. Nimafar, D. D. Ganji, Hydrothermal analysis of magneto hydrodynamic nanofluid flow between two parallel by AGM, Case Stud. Thermal Eng. 14, (2019) 100439.

[18] F. Ghomanjani, S. Shateyi, Alternative methods for solving nonlinear two-point boundary value problems, Open Phys. 16, No. 1 (2018) 371-374.

[19] A. K. Gupta, S. S. Ray, Comparison between HPM and OHAM for the soliton solutions of BoussinesqBurger equations, Comp. Fluids. 103 (2014) 34-41.

[20] S. Haq, M. Idrees, S. Islam, Application of optimal Homotopy asymptotic method to eighth order initial and boundary value problems, I. J. App. Maths Comp. 2, N0. 4 (2010) 73-80.

[21] H. D. Haustein, B. Kashi, Distortion of pipe-flow development by boundary layer growth and unconstrained inlet conditions, Phys. Fluids, 31, No.6 (2019) 063602.

[22] J. H. He, A coupling method of a homotopy technique and a perturbation technique for non-linear problems, I. j. non-linear mech. 35, No. 1 (2000) 37-43.

[23] N. Herisanu, V. Marinca, T. Dordea, G. Madescu, A new analytical approach to nonlinear vibration of an electrical machine, Pro. Romanian Acad. Series A-Maths. 9, No. 3 (2008) 229-236.

[24] M. K. Iqbal, M. Abbas, B. Zafar, New quartic B-spline approximation for numerical solution of third order singular boundary value problems, Punjab Univ J. math. 51, No. 5 (2020)

[25] M. K. Iqbal, M. Abbas, B. Zafar, New Quartic B-spline Approximations for Numerical Solution of Fourth Order Singular Boundary Value Problems, Punjab Univ J. math. 52, No. 3 (2020) 47-63.

[26] S. Javeed, A. Shabnam, D. Baleanu, An improved shooting technique for solving boundary value problems using higher order initial approximation algorithms, Punjab Univ. j. math. 51, No. 11 (2019) 101-113.

[27] H. D. Kasmaei, J. Rashidinia, Optimal Homotopy Asymptotic and Homotopy Perturbation Methods for Linear Mixed Volterra-Fredholm Integral Equations, Nev. Bilim Tekn. Der. 5, No. 2 (2016) 86-103.

[28] A. A. Khalid, M. Nawaz, Cubic B-spline solution of nonlinear sixth order boundary value problems, Punjab Univ. j. math. 50, No. 4 (2020).

[29] V. Marinca, R. D. Ene, B. Marinca, R. Negrea, Different approximations to the solution of upper-convected Maxwell fluid over a porous stretching plate, In Abs. App. Analysis. (2014).

[30] V. Marinca, N. Herisanu, Optimal homotopy perturbation method for strongly nonlinear differential equations, Non. Sci. Letters A. 1, No. 3 (2010) 273-280.

[31] V. Marinca, N. Herisanu, I. Nemes, Optimal homotopy asymptotic method with application to thin film flow, Open Phys. 6, No. 3 (2008) 648-653.

[32] V. Marinca, N. Herisanu, Application of the optimal homotopy asymptotic method for solving nonlinear equations arising in heat transfer, Int. Com. Heat Mass Trans. 35, No. 6 (2008) 710-715.

[33] S. T. Mohyud-Din, A. Yildirim, Solution of tenth and ninth-order boundary value problems by homotopy perturbation method, J. Korean Soc. Ind. App. Maths. 14, No.1 (2010) 17-27.

[34] K. Muhammad, T. Hayat, A. Alsaedi, OHAM analysis of fourth-grade nanomaterial in the presence of stagnation point and convective heat-mass conditions, W. Random Comp. Media. (2021) 1-17.

[35] M. Naeem, S. Muhammad, S. A. Hussain, Z. U. Din, L. Ali, Applying Homotopy Type Techniques to Higher Order Boundary Value Problems, J. Maths. 51, No. 2 (2019) 131-143.

[36] M. Naeem, S. Muhammad, S. A. Hussain, Z. U. Din, L. Ali, Applying homotopy type techniques to higher order boundary value problems, Punjab Univ. j. math. 51, No. 2 (2020).

[37] M. N. Naeem, Cubic Spline Solution of linear fourteenth order boundary value problems, Ceylon J. Sci. 47, No. 3 (2019) 253-261.

[38] R. Nawaz, M. N. Khalid, S. Islam, S. Yasin, Solution of tenth order boundary value problems using optimal homotopy asymptotic method (OHAM), Canadian J. Comp. Maths. Natural Sci. 1, No. 2 (2010) 37-54.

[39] P. Roul, A fast and accurate computational technique for efficient numerical solution of nonlinear singular boundary value problems, I. J. Comp. Maths. 96, No. 1 (2019) 51-72. 
[40] J. Saberi-Nadjafi, S. Zahmatkesh, Homotopy perturbation method (HPM) for solving higher order boundary value problems (BVP), App. Maths.Comp. Sci. 1 (2010) 199-224.

[41] A. Shafiq, F. Mebarek-Oudina,T. N. Sindhu, A. Abidi, A study of dual stratification on stagnation point Walters' B nanofluid flow via radiative Riga plate: a statistical approach, The Eur. Phys. J. Plus. 136, No. 4 (2021) 1-24.

[42] M. Qayyum, H. Khan, O. Khan, A New and Reliable Modification of Homotopy Perturbation Method, Punjab Univ. j. math. 48, No. 2 (2020).

[43] H. Ullah, R. Nawaz, S. Islam, M. Idrees, M. Fiza, The optimal homotopy asymptotic method with application to modified Kawahara equation, J. Ass. Arab Uni. Basic App. Sci. 18 (2015) 82-88.

[44] I. Ullah, M. T. Rahim, H. Khan, M. Qayyum, Analysis of various semi-numerical schemes for magnetohydrodynamic (MHD) squeezing fluid flow in porous medium, Prop. Power Res. 8, No. 1 (2019) 69-78. 